\title{
Perspectivas teóricas sobre salud y migración: determinantes sociales, transnacionalismo y vulnerabilidad estructural'
}

\section{Theoretical perspectives on health and migration: social determinants, transnationalism, and structural vulnerability}

\author{
Carlos Piñones-Rivera ${ }^{\mathrm{a}}$ \\ (iD) https://orcid.org/0000-0002-4771-3345 \\ E-mail: carlospinonesriveraळgmail.com \\ Nanette Liberona Concha ${ }^{b}$ \\ (D) https://orcid.org/0000-0002-0980-7519 \\ E-mail: nliberonacœgmail.com

\section{Sandra Leiva Gómez ${ }^{c}$} \\ (D) https://orcid.org/0000-0003-4883-6942 \\ E-mail: sandleivaœgmail.com \\ aUniversidad de Tarapacá. Escuela de Psicología y Filosofía. \\ Arica, AP, Chile. \\ bUniversidad de Tarapacá. Departamento de Antropología. \\ Arica, AP, Chile. \\ 'Universidad Arturo Prat. Instituto de Estudios Internacionales. \\ Iquique, $\mathrm{T}$, Chile.
}

\section{Correspondencia}

Carlos Daniel Piñones-Rivera

Universidad de Tarapacá. Av. 18 de septiembre, 2.222. Arica, AP, Chile. CP 1000000.

\section{Resumen}

El artículo analiza críticamente las tres aproximaciones teóricas contemporáneas más importantes en el abordaje del binomio saludmigración: el enfoque de determinantes sociales de la Organización Mundial de la Salud; los estudios sobre transnacionalismo y salud; y las propuestas sobre vulnerabilidad estructural. Se exponen las ideas centrales que les caracterizan y se presentan las principales críticas realizadas. Dado que la mayor parte de la literatura actual está siendo publicada en inglés, el artículo acerca de manera sintética algunas de las principales contribuciones en la materia al público hispanohablante, siendo el primer trabajo de este tipo que incluye la aproximación de la vulnerabilidad estructural. El análisis se apoyó en el software CAQDAS Nvivo, utilizando análisis de contenido sumariante, estructurante y explicativo. El trabajo enfatiza la importancia de los procesos de determinación estructural de la salud de los migrantes, y concluye abogando por un análisis de las convenciones científicas presentes en las perspectivas teóricas, en tanto estas tienen un impacto concreto en la salud de los migrantes, como fundamentos de políticas y como materia prima para el sentido común.

Palabras clave: Vulnerabilidad en Salud; Medicina Social; Inmigración; Determinantes Sociales de la Salud.

\footnotetext{
1 El presente artículo es resultado del proyecto Fondecyt Postdoctorado n 3180173 , "Itinerarios terapéuticos transfronterizos", del cual el Dr. Piñones Rivera es investigador responsable.
} 
This article critically analyzes the three major contemporary theoretical approaches in addressing health and migration: the social determinants of health approach of the World Health Organization; studies on transnationalism and health; and current proposals on structural vulnerability. To this end, the core ideas that characterize each of these approaches and the main criticisms made are presented. Given that most of the current literature is being published in English, this article summarizes some of the main contributions in the field for the Spanish-speaking public, constituting the first work of this type to include the structural vulnerability approach. The analysis was carried out with CAQDAS Nvivo, using summarizing, structuring, and explanatory content analysis. The article emphasizes the importance of the processes of structural determination of the health of migrants and concludes by advocating an analysis of the scientific conventions present in the theoretical perspectives, insofar as these have a concrete impact on the health of migrants, as policy's foundations and as raw material for common sense.

Keywords: Vulnerability in Health; Social Health; Immigration; Social Determinants of Health.
Las primeras publicaciones que abordan, en la literatura científica estadounidense, el problema de la relación entre salud y migración aparecen en 1912. Como ha sido una constante histórica (Abel, 2003), dichos trabajos sueldan una relación entre el migrante y las enfermedades infectocontagiosas, mostrando que "existe un vínculo entre la manera de imaginar una enfermedad y la de imaginar lo extranjero" (Sontag, 2003, p. 64). A partir de la advertencia de Sontag, nos interrogamos: ¿cómo es que hoy se está pensando la relación entre migración y salud? La pregunta es eminentemente práctica y resulta en el surgimiento de políticas, programas, proyectos, diagnósticos y prácticas de atención dirigidas a la salud de los migrantes. No es nuestra intención realizar un análisis sistemático de la literatura, puesto que esfuerzos en este sentido ya se han realizado (Castañeda et al., 2015; Villa-Torres et al., 2017; Viruell-Fuentes; Miranda; Abdulrahim, 2012). Nuestra contribución se focaliza en realizar un análisis crítico de lo que consideramos son las 3 aproximaciones principales desde las cuales hoy se piensa la salud de los migrantes: la de los determinantes sociales de la salud (DSS), la perspectiva del transnacionalismo aplicado a la salud, y las aproximaciones críticas, entre las que destaca el enfoque de la vulnerabilidad estructural. Una vez analizadas las dos primeras aproximaciones, el objetivo es presentar, en detalle, los aportes a los estudios de salud y migración del último enfoque.

Para esto, hemos hecho una revisión bibliográfica sobre salud y migración en los idiomas inglés y español. Las publicaciones que plasman las perspectivas escogidas fueron ingresadas al software CAQDAS Nvivo con cuya ayuda realizamos un análisis cualitativo de contenido integrando las tres técnicas definidas por Mayring (2004): por medio de un análisis de contenido sumariante, codificamos el material de manera de reducirlo a un texto corto manejable, que conserva su contenido esencial para nuestro problema; mediante un análisis de contenido estructurante, buscamos estructuras formales considerando las dimensiones del proceso salud/ enfermedad/atención que habían sido abordadas, enfatizadas y desatendidas de manera relativamente 
sistemática. Por último, por medio de un análisis de contenido explicativo, buscamos construir un análisis coherente del material, involucrando material de contexto estrecho y amplio. Dicho análisis nos llevó a identificar a estas tres como las aproximaciones más significativas en el debate contemporáneo sobre el binomio salud-migración.

Dado que buena parte de la literatura analizada está escrita en inglés, una contribución del presente manuscrito es acercar a la comunidad hispanohablante parte de dicha literatura. Más aun, queremos contribuir con el progresivo fortalecimiento de las aproximaciones basadas en la medicina social, pues nos parece necesario en momentos en los cuales tanto el multiculturalismo neoliberal como el racismo antinmigrante adquieren fuerza al producir entornos sociopolíticos patogénicos en general y en específico para los migrantes.

\section{Los determinantes sociales de la salud (DSS)}

La perspectiva de los DSS es probablemente la que más amplio consenso tiene dentro de los abordajes sobre el binomio salud y migración. Parte de dicho consenso se basa en que ella se presenta en oposición a las perspectivas reduccionistas biologicistas, por lo que en sus planteamientos se pueden ver reflejadas aproximaciones muy diversas, que enfatizan los aspectos psicosociales, culturales o, incluso, como hacen las perspectivas críticas, los procesos de determinación económico-política.

La perspectiva de los determinantes sociales está desde el año 2009 en el centro de las propuestas consagradas en la Organización Mundial de la Salud (OMS). No obstante, su historia se remonta mucho más atrás y se confunde -en un sentido específico-con algunas de las propuestas desarrolladas en los años 70 en el fragor de las discusiones propias de Alma Ata, pero -en un sentido más amplio- con las discusiones y propuestas que han emergido de la larga tradición de la medicina social (Solar; Irwin, 2006).

La definición canónica de los determinantes sociales los conceptualiza como

las circunstancias en que las personas nacen, crecen, viven, trabajan y envejecen, incluido el sistema de salud. Esas circunstancias son el resultado de la distribución del dinero, el poder y los recursos a nivel mundial, nacional y local, que depende a su vez de las políticas adoptadas. (OMS, 2018)

En el actual abordaje de la OMS respecto de la salud de los migrantes, el modelo de los determinantes sociales es una de las piezas claves, junto con un esquema del "ciclo migratorio". Así el panorama incluye a las vulnerabilidades y resiliencias que surgen de los determinantes sociales a lo largo de todo el ciclo migratorio (origen, tránsito, destino y retorno) en un marco de acciones que tributan a la Agenda 2030 de las Naciones Unidas (WHO, 2017). Como se observa el modelo presenta una imagen bastante amplia y compleja de los factores en juego e incluye tanto los elementos productores de enfermedad como los protectores.

No obstante, esta definición aparentemente neutral y abarcativa deja de parecerlo si se mira a la luz de la historia de la discusión dada en el espacio de la medicina social. En él, los procesos de lucha contra la mercantilización de la salud y la destrucción propia de la acumulación capitalista llevaron a desarrollar una mirada crítica de la aproximación clínica como vía para mejorar la salud de los colectivos, en tanto ella invisibiliza "las relaciones de determinación generadas por el sistema económico de acumulación de capital, las relaciones de inequidad que lo reproducen y la destrucción de la naturaleza” (Breilh, 2013, p. 14).

El devenir hegemónico de la concepción de los DSS resulta de una institucionalización parcial de esta concepción crítica, operada en el seno de la OMS y que tiene como correlato la progresiva influencia del Banco Mundial en los ámbitos de competencia de la OMS (Irwin; Scali, 2007). En dicha institucionalización, la crítica al rol del modo de producción capitalista se traduce en una alusión inespecífica al poder, plasmada en las siguientes líneas: "Esas circunstancias son el resultado de la distribución del dinero, el poder y los recursos a nivel mundial, nacional y local, que depende a su vez de las políticas adoptadas" (OMS, 2018).

Un buen ejemplo de cómo se puede llegar a deformar el espíritu de las iniciativas contrahegemónicas en salud lo constituye la versión chilena de los DSS. En ella se sostiene que 
los determinantes sociales de la salud se entienden como las condiciones sociales en que las personas viven y trabajan, que impactan sobre la salud [...] Los determinantes sociales objetos de políticas públicas son aquellos susceptibles de modificación mediante intervenciones efectivas. (Chile, 2019)

Como se observa, tanto la crítica al sistema económico de acumulación de capital como las relaciones de inequidad que lo reproducen y la destrucción de la naturaleza desaparecen de la definición chilena, o bien se traducen por medio del inocuo calificativo de "social" (Piñones-Rivera; Mansilla Agüero; Arancibia Campos, 2017).

Otro ejemplo paradigmático del uso de los DSS son los informes realizados por el equipo de Báltica Cabieses (Cabieses; Bernales; McIntyre, 2017; Cabieses et al., 2016; Cabieses et al., 2017), entre los cuales se sitúa su análisis de la vulnerabilidad social de la población migrante de la comuna de Iquique (Tarapacá, Chile) en términos de las "condiciones sociales de la salud": pobreza, vivienda, delitos (VIF), calidad de vida urbana, microtráfico de drogas, delincuencia y prostitución.

Si bien pareciera que esta aproximación constituye un buen remedio contra el reduccionismo propio del biologicismo biomédico, la forma como se presentan los datos sirve para reforzar elementos racistas presentes en el imaginario chileno (Tijoux; Córdova, 2015; Tijoux; Palominos, 2015). Por ejemplo, al analizar la situación de la vivienda y, en particular, el hacinamiento y las malas condiciones de esta (Cabieses; Bernales; McIntyre, 2017) no se relacionan dichas condiciones con la dinámica de segregación racial que Contreras Gatica, Ala-Louko y Labbé (2015) han denominado "acceso exclusionario y racista a la vivienda”, que empuja a los migrantes a los barrios en que imperan esas condiciones. Menos aún se nos muestra cómo tanto el hacinamiento, el empuje a las tomas y, en general la vida en "malas condiciones" se relacionan con la mercantilización neoliberal del derecho a la vivienda (Imilan, 2016).

En el ejemplo, es patente que cuando no se analiza la producción socioeconómica y la política neoliberal de las condiciones que producen la realidad social de los migrantes en Chile, lo que aparece como una deseable descripción "objetiva” de la "vulnerabilidad social" de los migrantes ofrece elementos objetivados que refuerzan los prejuicios racistas existentes, así como la idea de que pobreza, hacinamiento, delincuencia y drogas constituyen el lugar social connatural a los migrantes. ${ }^{2}$ Cuestión denunciada por Eduardo Bonilla-Silva: "esta es la forma central en que los estudiosos contemporáneos contribuyen a la propagación de interpretaciones racistas de la desigualdad racial. Al no destacar las dinámicas sociales que producen estas diferencias raciales, estos estudiosos ayudan a reforzar el orden racial" (Bonilla-Silva, 2006, p. 8, traducción nuestra).

Notemos, por último, que la aproximación de los DSS reúne procesos muy disímiles respecto al impacto negativo en la salud, como el hacinamiento, los problemas de salud ambiental, la pobreza multidimensional y los procesos biológicos, integrándolos en un cuadro que asume que no existe ningún orden estructurante en la multiplicidad de causas (Breilh, 2013; Krieger, 1994). Esto es justamente lo contrario al esfuerzo inicial tras la discusión de los procesos de determinación social y la subsunción (Breilh, 1994, 2013; Franco et al., 1991). En efecto, en una comparación de la epidemiología social latinoamericana y la anglosajona se señala:

Mientras que los DSS-a [anglosajona] entienden que los resultados en salud están relacionados con un contexto social donde la posición social del individuo genera exposición y vulnerabilidad diferencial que explica la distribución de las inequidades en salud, pero invisibiliza las fuerzas en tensión y relaciones de poder de la sociedad; la DSS-b [latinoamericana] releva las relaciones de poder y exalta las dinámicas

2 Un elemento interesante es que, en general, en estos análisis no se destacan los determinantes sociales que pueden jugar en favor de la salud de los migrantes, ni su contribución a la salud de los países de destino, ya sea a través de los saberes de autoatención que vehiculizan, o de los especialistas de salud de los saberes hegemónicos o subalternizados (PIÑONES-RIVERA, C.; MUÑOZ HENRÍQUEZ, W.; LIBERONA CONCHA, N. Te mueves o te mueres: la movilidad del saber médico andino en la triple frontera Bolivia, Chile y Perú. In: FERRARI, M. et al. Fronteira, território e ambiente: diálogos entre América Latina e Europa. Cascavel: Edunioeste. No prelo). 
de acumulación de capital como esenciales para entender la determinación social de los procesos saludenfermedad que conllevan los patrones de trabajo y de consumo, las falencias de los soportes sociales y las formas de cultura que conducen a formas malsanas de vivir y llevan a los individuos a padecer la enfermedad de forma diferencial, según clase social, género o etnia. (Morales-Borrero et al., 2013, p. 80o-8o1)

\section{Transnacionalismo y salud}

La segunda aproximación escogida es la del transnacionalismo, que podemos caracterizar, aunque con el riesgo de simplificarla, como una perspectiva teórica que busca superar la linealidad y unidireccionalidad propia de los estudios migratorios previos, cuyos análisis estaban centrados en los conceptos de asimilación y aculturación, así como aquejados del "nacionalismo metodológico" (Glick Schiller, 2009; Wimmer; Glick Schiller, 2002, 2003). Para esto, piensa las relaciones, lazos e interacciones sociales, culturales, económicas y políticas que se establecen a través de las fronteras, mostrando que los migrantes no solo no dejan su cultura atrás, sino que ayudan a transformar los lugares de origen y de llegada, en virtud de las relaciones y dinámicas transfronterizas que establecen. De esta manera, el transnacionalismo ha mostrado la emergencia de "espacios sociales transnacionales" (Villa-Torres et al., 2017).

La perspectiva del transnacionalismo ha sido recientemente aplicada a la investigación en salud. A nivel internacional explícitamente lo han trabajado autores como Baldassar (2014), Grineski (2011), Horton (2013), Madden (2015) y Villa-Torres et al. (2017). No obstante, existe una extensa literatura respecto del turismo médico (Connell, 2015; Crush; Chikanda, 2014; Ormond; Sulianti, 2017), que también puede ser incorporada para pensar las relaciones transnacionales en salud, así como los ya clásicos trabajos de Connell (2015) y Bell et al. (2015). En Chile, Liberona Concha, Tapia Ladino y Contreras (2017) han examinado la movilidad por salud transfronteriza entre Arica y Tacna desde un análisis crítico del concepto de turismo médico, considerando la mercantilización de los sistemas de salud.
Algunas de las conclusiones importantes que se han establecido en el campo del transnacionalismo y salud son (Villa-Torres et al., 2017):

- La salud y las conductas relacionadas están influenciadas por el transnacionalismo, existiendo la necesidad de indagar sistemáticamente cómo las prácticas de salud de los migrantes se encuentran incorporadas en el proceso de migración trasnacional.

- En términos espaciales, se ha dado cuenta de la existencia de "paisajes terapéuticos transnacionales”. En otras palabras, el proceso de curación se desarrolla en el espacio mediante la negociación entre factores territoriales, individuales y sociales, poniendo el énfasis en el carácter transnacional, es decir, mostrando cómo dichos paisajes superan las fronteras nacionales, dibujando nuevas unidades territoriales funcionales.

- Se ha valorizado que los migrantes poseen un "capital cultural transnacional" (Grineski, 2011), que incide en la salud. Este incluye el saber sobre el papeleo para cruzar fronteras, información, derivaciones, telediagnóstico, acceso a medicamentos (desde lugares de origen y destino), acceso a curadores tradicionales y uso de servicios de salud formales e informales en los países de origen y destino.

A lo anterior podríamos añadir las importantes contribuciones de Thomas Faist sobre las condiciones institucionales para la integración de los migrantes en los estados de bienestar (Dörr; Faist, 1997), así como sus recientes trabajos referidos a la protección social informal transnacional, en que visibiliza la contribución que las estrategias informales realizan al enfrentamiento de los riesgos asociados a la producción (p. ej. trabajo) y reproducción (p. ej. cuidado) (Faist et al., 2015), a la vez que problematiza la producción y reproducción de la inequidad social que tiene lugar por medio de dichas estrategias. Por último, Faist ha dado una nueva definición de transnacionalidad como marcador de heterogeneidad, en el cruce del enfoque transnacional y el de la interseccionalidad, que deja 
de definirla como una característica dicotómica para verla como una variable (Faist et al., 2015).

De los muchos aspectos que podríamos analizar de este enfoque, nos queremos centrar en lo que respecta al uso del concepto de "capital cultural". Como es sabido, el concepto es tomado de Bourdieu para superar una aproximación sociológica que se basaba de manera restrictiva en el capital económico. Así es definido como:

Un tipo único de capital cultural que otorga el poder para lograr fines a través de las fronteras. A diferencia de ser inculcado en una única cultura (por ejemplo, estadounidense o mexicana), el Capital Cultural Transnacional es el poder de conseguir lo que uno percibe como lo más útil, en un campo de atención de la salud que supera las fronteras. (Grineski, 2011, p. 258, traducción nuestra)

Cómo se aplica prácticamente en la comprensión del binomio salud-migración? Para nuestro análisis crítico tomaremos el ejemplo de Grineski quien establece que para los padres que buscaban atención médica a sus niños asmáticos en Phoenix (Arizona) eran ventajas clave del capital cultural transnacional: "hablar inglés, trabajar en el campo de la salud, tener un título universitario y nacer en los Estados Unidos" (Grineski, 2011, p. 258, traducción nuestra).

Llama la atención el supuesto de que el estándar del "capital cultural ventajoso" para abordar los problemas de salud coincida con el estándar de la asimilación (al saber biomédico, la lengua, la escolaridad y la nacionalidad propias de los Estados Unidos). En la misma vertiente teórica, otros autores han estudiado el encuentro médico-paciente y encontraron que las relaciones de salud tienen más éxito cuando, tanto los pacientes como los médicos, adoptan un conjunto de habilidades culturales que incluyen competencias verbales y no verbales, estilos de interacción y actitudes, que Shim (2010) ha denominado "capital cultural de salud".

Queremos detenernos a reflexionar sobre ¿qué tipo de sentidos puede vehiculizar esta aproximación?, ¿en qué tipo de habilidades o capital cultural se está pensando? Grineski (2011, p. 258, traducción nuestra) nos señala:
Los de la clase social inferior, y específicamente los inmigrantes, tenían menos probabilidades de poseer estos recursos culturales, lo que les dificultaba administrar con éxito el asma de sus hijos; mientras intentaban desplegar capital social para compensar su falta de recursos económicos y capital cultural, no fue tan útil en el campo de la salud.

Es decir, el mantenimiento de los saberes culturales propios es interpretado como una fuente de dificultades. A partir del supuesto de la supremacía del saber biomédico, se da por sentado que la clave del abordaje de los problemas de salud radica en la asimilación con el saber biomédico, desconociendo que el universo del "capital cultural" es un conjunto mucho más amplio, en el cual, con justa razón, se pueden incluir los saberes indígenas en el abordaje de la salud, o formas de sanidad espiritual propias de las múltiples denominaciones cristianas, por citar algunas de las más relevantes en los estudios de salud intercultural con población indígena. Aquí notamos una diferencia importante con la tradición de la Antropología Médica Crítica Latinoamericana, que desde los años 70 ha subrayado la importancia del pluralismo médico como forma de visibilizar la coexistencia de saberes médicos, desnaturalizando la hegemonía de la cultura biomédica.

Si bien el transnacionalismo ha constituido una importante contribución al abordaje de la salud de los migrantes, siendo recientemente identificada como una de las principales contribuciones de la teoría social para la toma de decisiones en salud pública respecto de los migrantes (Cabieses; Galvez; Ajraz, 2018), compartimos una de las principales críticas que se ha realizado a los estudios de transnacionalismo y salud, en confluencia con lo que hemos problematizado en el enfoque de los DSS:

A nivel estructural, la capacidad de promulgar esta transnacionalidad y mover y movilizar recursos a través de las fronteras requiere un análisis de las políticas que se intersectan con las políticas de salud, como las políticas de inmigración, trabajo y bienestar social [...]. En definitiva, lo que observamos con esta revisión es que muchas prácticas de salud transnacionales no abordan las causas fundamentales de las inequidades en salud, 
sino que rondan en torno a la falta de protecciones sociales transnacionales. (Villa-Torres et al., 2017, p. 77, traducción nuestra)

Esta dificultad para abordar lo estructural ha sido planteada ya por otros autores no solo en relación al campo de la salud, sino en la comprensión de los procesos migratorios en general. De esta manera, Márquez Covarrubias (2012, p. 318-319) señala:

El punto central para esta perspectiva lo constituye el entramado de relaciones socioculturales que tensan los migrantes entre sí y sus familiares, lo cual permite enlazar lugares de destino y origen [...]. En esa misma pauta, enaltecen la agencia de los migrantes, que contempla desde la organización social de las migraciones, las redes sociales, las organizaciones de migrantes y sus vínculos con sus lugares de origen. Al enaltecer la agencia, pierde de vista la dimensión estructural y estratégica, es decir, la dimensión política e institucional donde cohabitan el poder político, representado por el Estado, y el poder económico, representado por el capital.

\section{Aproximaciones críticas: vulnerabilidad estructural}

Una de las aproximaciones contemporáneas más vigorosas en el abordaje del binomio salud-migración es el de la vulnerabilidad estructural. La noción es tomada del antropólogo mexicano Daniel Hernández Rosete (Piñones-Rivera; Quesada; Holmes, 2019), pero surge como concepto el año 2011 en textos de Quesada, Hart y Bourgois (2011) y Holmes (2011). En él se puede ver confluir una serie de aproximaciones críticas en el campo de la Antropología Médica Crítica, la Medicina Social, la Epidemiología Crítica o la Salud Pública, que han mostrado cómo la estructura social (Stonington et al., 2018) impone riesgos y constricciones específicas a la salud individual y colectiva. A diferencia de lo señalado en las aproximaciones previas, este enfoque señala enfáticamente que el problema de la salud de los migrantes debe ser abordado considerando, en primer lugar, el problema de la estructura económica y política, o el modo de producción. ${ }^{3}$

En este terreno, una importante contribución desde las Ciencias Sociales anglosajonas ha venido del concepto de violencia estructural. Como tempranamente conceptualizó Galtung, es aquel tipo de violencia en que no es posible identificar de manera directa un agente, pues la violencia está entramada en la estructura de la sociedad y en la misma existencia de posiciones jerarquizadas. Desde esta matriz surge el concepto de vulnerabilidad estructural como herramienta para comprender cómo los mecanismos de la violencia estructural afectan el cuerpo, la salud, la enfermedad e interfieren en la atención, cristalizando vulnerabilidades específicas (Holmes, 2013b). En esta mirada, se pone énfasis en que son las estructuras socioeconómicas y políticas las que producen y organizan los riesgos, el daño y el sufrimiento (Holmes, 2011), pues estos están determinados por la posición que ocupa la persona en el ordenamiento jerárquico (Quesada; Hart; Bourgois, 2011), ya que es dicha posición la que define las opciones de vida (Holmes, 2011) y es donde operan, de manera conjugada, distintas formas de opresión: de clase, raza/etnicidad, género, estatus legal migratorio, nacionalidad, entre los más importantes (Bourgois, 1988).

\section{El lugar de la migración en la estructura económica capitalista y el impacto que tiene en la salud de los migrantes}

Basándose en Burawoy (1976), Holmes (2013a) muestra cómo los sistemas de trabajo migrante son caracterizados por establecer una separación temporal y física entre los procesos de producción y los de reproducción de la fuerza de trabajo. El migrante puede sobrevivir con bajos salarios solo porque la educación, los cuidados de salud y otros son provistos en el país de origen. Así el país de "acogida" externaliza el costo de dichos procesos. La situación total encierra una contradicción económico-política, pues mientras, por un lado, la situación económica

\footnotetext{
3 No es un secreto que esta aproximación se nutre extensamente de las contribuciones de la Medicina Social Latinoamericana/Salud Colectiva. Cfr. Piñones-Rivera, Quesada y Holmes (2019).
} 
requiere de la fuerza de trabajo migrante, por otro lado, la estructura política le priva de los derechos básicos de la ciudadanía, limitando severamente su poder de injerencia en el trabajo. De esta manera, el sistema de trabajo migrante descansa en la dificultad del migrante de influenciar las instituciones y estructuras que lo subordinan, por lo que se produce el efecto de una máxima explotación de la fuerza de trabajo, con todo el sufrimiento y la carga de enfermedad que eso supone (Holmes, 2013a).

Esto hace que sea posible describir una jerarquía del sufrimiento, pues el trabajo está segregado según jerarquías étnico-ciudadano-laborales, que exponen a daños, riesgos y recursos de salud de manera diferencial. Por ejemplo, mientras la indigeneidad percibida define la ocupación, la posición que se ocupa en el trabajo dependerá mucho del estatus migratorio y de la ciudadanía (Holmes, 2013a). La opresión conjugada de este y otros procesos de determinación hacen que los migrantes sean probablemente los más vulnerables en la estructura jerárquica, y dicha vulnerabilidad es funcional al sistema de producción capitalista en la medida que le asegura mayor estabilidad en la provisión de la fuerza de trabajo, mayor explotación -productividady garantiza una disminución, al mínimo, de la capacidad de influenciar las condiciones de su trabajo (Holmes, 2007, p. 48-49)

\section{Cómo las jerarquías sociales son naturalizadas por medio de la violencia simbólica}

Otro de los aspectos significativos son los procesos de naturalización que están tanto a la base de la reproducción de las jerarquías sociales, como de la incomprensión del sufrimiento de los migrantes por la sociedad en general y los trabajadores de la salud. Para esto, se ha recurrido al concepto de violencia simbólica de Bourdieu, según el cual la aceptación de la dominación, con toda la violencia que supone, se produce porque "cada grupo percibe como natural no solo su propia posición, sino también la del otro grupo, dentro de la jerarquía social” (Holmes, 2016, p. 75). Es decir, existe un desconocimiento (misrecognition) fundacional, tanto respecto de la naturaleza jerárquica del funcionamiento social como del rol que jugamos en el proceso mismo de su reproducción.

Así se ha mostrado cómo la naturalización impacta en la salud de los migrantes por distintas vías:

a. La naturalización de la explotación laboral: Holmes (2016, p. 217) muestra cómo el lugar que ocupan los migrantes mexicanos indígenas en la jerarquía social y el tipo de explotación que sufren es naturalizado mediante ciertas expresiones como "A los Oaxaqueños les gusta trabajar agachado". Desde el desconocimiento de la realidad social y las condiciones que los llevan a trabajar en dichos contextos, se atribuye equívocamente la correlación entre la posición social étnicamente definida y el tipo de trabajo, a ciertas características corporales: "Los oaxaqueños son demasiado bajos para alcanzar las manzanas, son muy lentos... Tienen que usar escaleras mucho más que otros" (Holmes, 2016, p. 217). Las percepciones, así como los significados asociados, constituyen las formas a través de las cuales se materializa la violencia simbólica, fijando al migrante en una posición social en la cual supuestamente merece estar, estableciendo una relación de autoevidencia entre el cuerpo de los migrantes y el tipo de trabajo que realizan (Holmes, 2016).

b. La naturalización de la posición social por internalización: Dado que la violencia simbólica organiza las percepciones y prácticas tanto de los dominantes como de los dominados, se intenta comprender las distintas negociaciones identitarias. Por un lado, se muestra cómo se identifican conflictivamente con ciertos estigmas asociados a la migración (Organista et al., 2013; Quesada, 2011; Quesada et al., 2014), por otro lado, también muestran el proceso

\footnotetext{
4 Algo similar se puede encontrar en Fassin (1999, 2000), quien analiza las disparidades en el acceso a la salud de los inmigrantes y extranjeros, considerando que su salud existe en la relación históricamente construida por actores sociales, planteando los problemas de inequidad en función de las jerarquías sociales, resultantes de la condición colonial (Fassin, 2004).
} 
más sutil, según el cual cultivan un orgullo identitario que, por una parte, les ayuda a enfrentar condiciones laborales extremas "Los pesticidas afectan solo a los estadounidenses blancos (gabachos) porque sus cuerpos son delicados y débiles [...] Nosotros, los triquis, somos fuertes y aguantamos" (Holmes, 2016, p. 220); pero a la vez, al basarse en las distinciones propias del juego de la violencia simbólica, refleja la internalización de las jerarquías sociales y termina reforzando la explotación de la que son objeto. El biomédico, cuya posición en la jerarquía social es incomparable a la del trabajador migrante, es también afectado por la ubicuidad de la violencia estructural, según se refleja en el doble vínculo al cual está sometido el profesional de la salud en contextos capitalistas; por un lado, dar cumplimiento a las tareas burocráticas y estadísticas, por el otro, debe examinar, entrevistar y organizar un plan de atención en el tiempo, y todo en 15 minutos (Holmes, 2016).

c. La naturalización de las enfermedades atribuidas al descuido individual (individualismo biomédico): por medio de la ideología biologicista y del individualismo característicos de la mirada médica, casi indefectiblemente identifica el lugar de la responsabilidad de la enfermedad en el paciente, culpabilizando a la víctima (Ryan, 1976). Cuando al individualismo biomédico se le añade el supuesto culturalista de que sería en virtud de ciertas características culturales que los pacientes enferman, se configura una nueva forma de racismo, el de las competencias culturales (Pon, 2009).

Estos procesos de naturalización forman parte de la violencia simbólica hacia los migrantes y producen importantes efectos en su salud. La vulnerabilidad estructural permite comprender por qué las inequidades descritas se mantienen incuestionadas y no-desafiadas, tanto por los migrantes mismos como por el personal de salud y por la sociedad en general.

\section{Cómo el racismo estructural afecta la salud de los migrantes}

Por último, esta aproximación incorpora el desplazamiento que se ha producido en el abordaje del racismo, desde el racismo individual o interaccional, hasta el racismo institucional (Krieger, 2014), sistémico (Castle, 2019; Feagin, 2006; Feagin; Bennefield, 2014) o más propiamente estructural. En este viraje, el enfoque se nutre de las aproximaciones a la salud pública planteadas a partir de la teoría crítica de la raza (Airhihenbuwa; Ford, 2018; Ford; Airhihenbuwa, 2010a, 2010b; Hicken et al., 2018; Madden, 2015; Metzl; Roberts, 2014).

El racismo estructural se define como "los sistemas de nivel macro, las fuerzas sociales, las instituciones, las ideologías y los procesos que interactúan entre sí para generar y reforzar las inequidades entre los grupos raciales y étnicos" (Gee; Ford, 2011, p. 3, traducción nuestra). El concepto enfatiza los niveles socioecológicos más influyentes en los cuales el racismo puede afectar las desigualdades raciales y étnicas de salud. Esclarece que los mecanismos estructurales no requieren las acciones o la intención de los individuos, pues en tanto causas fundamentales (Phelan; Link, 2015) reconstituyen constantemente las condiciones necesarias para garantizar su perpetuación. Al punto que, si se eliminara por completo la discriminación interpersonal, las desigualdades raciales probablemente permanecerían sin cambios, debido a la persistencia del racismo estructural (Gee; Ford, 2011).

Es entonces este racismo, con todas sus sutilezas, operando en los espacios más disímiles y con sus profundas raíces históricas lo que es puesto como foco de análisis en relación con los procesos de determinación del sufrimiento de los migrantes. Mientras que la literatura previa plantea que el racismo es un factor que afecta la salud, constituyendo una barrera a la atención, el racismo estructural se focaliza en el impacto que tienen en la salud las políticas de inmigración, las políticas públicas en general, los procesos de segregación residencial (Williams; Collins, 2001), la segregación étnica de los espacios de trabajo, 
pero también los modelos teóricos desde los cuales se piensan los problemas de salud o la producción de conocimiento científico cuando opera en una lógica racializada. ${ }^{5}$

En un esfuerzo por sistematizar una aproximación de salud pública capaz de transformar la praxis y la investigación desde las cuales se aborda el racismo en salud, se han desarrollado aproximaciones específicas destacando las de Ford y Airhihenbuwa (2010a, 2010b). Sus trabajos definieron 4 áreas en las que se focaliza la intervención (Figura 1) y 10 principios constitutivos de cualquier investigación basada en la teoría crítica de la raza (Tabla 1).

\section{Figura I - Conciencia de racialización, los cuatro focos y diez principios relacionados}

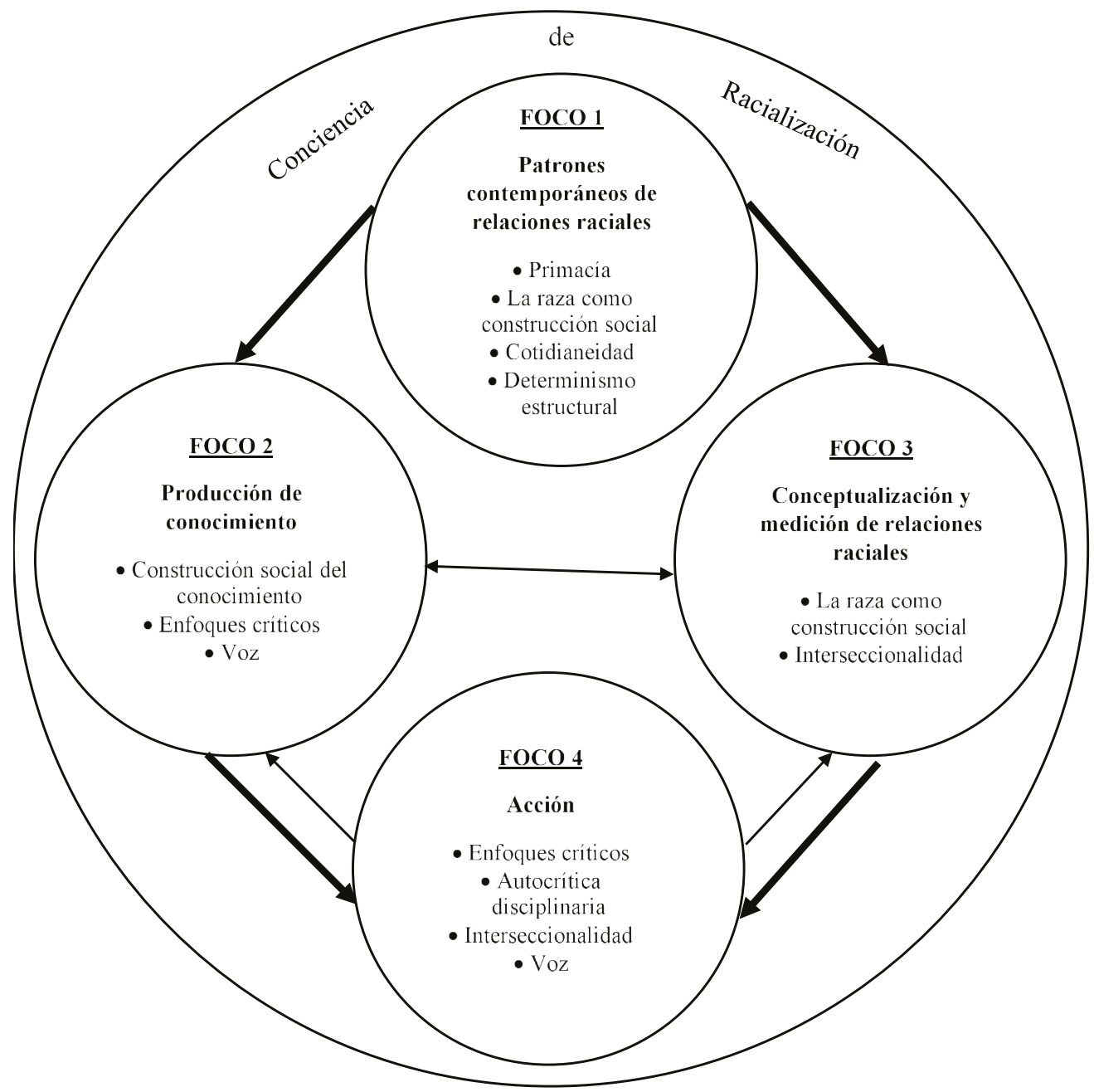

Fuente: Ford y Airhihenbuwa (2010b, p. 1391, traducción nuestra)

5 Un planteamiento similar encontramos en Fassin (2004, p. 295), quien desde una "antropología política de la salud" apela a una necesaria revisión de la "moral política” de los países de destino. Para esto, asocia los temas de salud pública a los de las políticas de inmigración al destacar aspectos como la construcción de la diferencia en el campo de la salud y la cuestión del acceso de los migrantes a la ciudadanía (Fassin, 200o). 
Tabla I - Principios de CRSP y focos relacionados

\begin{tabular}{|c|c|c|c|c|}
\hline Principios & $\begin{array}{l}\text { Focos } \\
\text { relacionados }\end{array}$ & Definición & $\begin{array}{l}\text { Aproximaciones } \\
\text { convencionales }\end{array}$ & $\begin{array}{l}\text { Aproximación crítica de } \\
\text { la raza en salud pública }\end{array}$ \\
\hline $\begin{array}{l}\text { I. Conciencia de la } \\
\text { racialización }\end{array}$ & Todos & $\begin{array}{l}\text { Profunda conciencia } \\
\text { de la posición } \\
\text { racializada de } \\
\text { uno; Conciencia } \\
\text { de los procesos } \\
\text { de estratificación } \\
\text { racial que operan en } \\
\text { contextos daltónicos. }\end{array}$ & $\begin{array}{l}\text { "Colorblindness- } \\
\text { belief": Tendencia } \\
\text { a atribuir las } \\
\text { desigualdades } \\
\text { raciales a factores } \\
\text { no-raciales ( } p . \text { ej. } \\
\text { NSE), considerando } \\
\text { irrelevante el racismo. }\end{array}$ & $\begin{array}{l}\text { Un investigador aclara } \\
\text { sus prejuicios raciales } \\
\text { antes de comenzar la } \\
\text { investigación dentro } \\
\text { de una comunidad } \\
\text { diversa. }\end{array}$ \\
\hline $\begin{array}{l}\text { 2. Primacía de la } \\
\text { racialización }\end{array}$ & $\begin{array}{l}\text { Racialización } \\
\text { contemporánea }\end{array}$ & $\begin{array}{l}\text { La contribución } \\
\text { fundamental de la } \\
\text { estratificación racial } \\
\text { a los problemas } \\
\text { sociales; El foco } \\
\text { central de la } \\
\text { investigación desde la } \\
\text { TCR para explicar los } \\
\text { fenómenos raciales. }\end{array}$ & $\begin{array}{l}\text { Tendencia a atribuir } \\
\text { efectos a la raza } \\
\text { en lugar de la } \\
\text { racialización o al } \\
\text { racismo. }\end{array}$ & $\begin{array}{l}\text { Un estudio sobre las } \\
\text { características del } \\
\text { vecindario incluye } \\
\text { factores hipotetizados } \\
\text { para reflejar el racismo } \\
\text { estructural. }\end{array}$ \\
\hline $\begin{array}{l}\text { 3. Raza como } \\
\text { constructo social }\end{array}$ & $\begin{array}{l}\text { Racialización } \\
\text { contemporánea, } \\
\text { conceptualización } \\
\text { y mediciones }\end{array}$ & $\begin{array}{l}\text { Significado que } \\
\text { deriva de las fuerzas } \\
\text { sociales, políticase } \\
\text { históricas. }\end{array}$ & $\begin{array}{l}\text { Determinismo biológico: } \\
\text { la creencia de que la } \\
\text { raza es significativa } \\
\text { porque proporciona } \\
\text { información sobre la } \\
\text { biología y las tendencias } \\
\text { del individuo. }\end{array}$ & $\begin{array}{l}\text { Un estudio aborda } \\
\text { la raza no como un } \\
\text { factor de riesgo sino } \\
\text { para identificar una } \\
\text { población en riesgo de } \\
\text { exposiciones específicas } \\
\text { al racismo. }\end{array}$ \\
\hline $\begin{array}{l}\text { 4. Cotidianeidad } \\
\text { del racismo }\end{array}$ & $\begin{array}{l}\text { Racialización } \\
\text { contemporánea }\end{array}$ & $\begin{array}{l}\text { El racismo está } \\
\text { incrustado en el tejido } \\
\text { social de la sociedad. }\end{array}$ & $\begin{array}{l}\text { Excepcionalismo racial: } \\
\text { define el racismo } \\
\text { como incidentes } \\
\text { raros, discretos y } \\
\text { abiertamente atroces. }\end{array}$ & $\begin{array}{l}\text { Un estudio sobre el } \\
\text { racismo y la salud } \\
\text { operacionaliza el } \\
\text { racismo como exposición } \\
\text { rutinaria (por ejemplo, } \\
\text { que se siga al hacer } \\
\text { compras). }\end{array}$ \\
\hline $\begin{array}{l}\text { 5. Determinismo } \\
\text { Estructural }\end{array}$ & $\begin{array}{l}\text { Racialización } \\
\text { contemporánea }\end{array}$ & $\begin{array}{l}\text { El rol fundamental } \\
\text { de las fuerzas de } \\
\text { nivel macro en la } \\
\text { producción y el } \\
\text { mantenimiento de } \\
\text { las desigualdades } \\
\text { en el tiempo y los } \\
\text { contextos; la tendencia } \\
\text { de los miembros de los } \\
\text { grupos dominantes } \\
\text { e instituciones para } \\
\text { tomar decisiones o } \\
\text { acciones que preserven } \\
\text { las jerarquías de poder } \\
\text { existentes }\end{array}$ & $\begin{array}{l}\text { Destaca los factores } \\
\text { individuales o } \\
\text { interpersonales. }\end{array}$ & $\begin{array}{l}\text { Un estudio multinivel } \\
\text { considera factores } \\
\text { políticos que } \\
\text { pueden promover } \\
\text { la segregación } \\
\text { residencial. }\end{array}$ \\
\hline
\end{tabular}


Tabla I-Continuación

\begin{tabular}{|c|c|c|c|c|}
\hline Principios & $\begin{array}{l}\text { Focos } \\
\text { relacionados }\end{array}$ & Definición & $\begin{array}{l}\text { Aproximaciones } \\
\text { convencionales }\end{array}$ & $\begin{array}{l}\text { Aproximación crítica de } \\
\text { la raza en salud pública }\end{array}$ \\
\hline $\begin{array}{l}\text { 6. Construcción } \\
\text { social del } \\
\text { conocimiento }\end{array}$ & $\begin{array}{l}\text { Producción de } \\
\text { conocimiento }\end{array}$ & $\begin{array}{l}\text { La afirmación de } \\
\text { que el conocimiento } \\
\text { establecido dentro de } \\
\text { una disciplina puede } \\
\text { reevaluarse utilizando } \\
\text { modos de análisis } \\
\text { antirracistas. }\end{array}$ & $\begin{array}{l}\text { La creencia de que } \\
\text { la investigación } \\
\text { empírica realizada } \\
\text { adecuadamente es } \\
\text { impermeable a las } \\
\text { influencias sociales. }\end{array}$ & $\begin{array}{l}\text { Una revisión de la } \\
\text { literatura relacionada } \\
\text { con las disparidades } \\
\text { compara los artículos } \\
\text { publicados en revistas } \\
\text { minoritarias frente a las } \\
\text { mayoritarias. }\end{array}$ \\
\hline $\begin{array}{l}\text { 7. Aproximaciones } \\
\text { Críticas }\end{array}$ & $\begin{array}{l}\text { Producción de } \\
\text { conocimiento, } \\
\text { Acción }\end{array}$ & $\begin{array}{l}\text { Cavar bajo la } \\
\text { superficie; Desarrollar } \\
\text { un entendimiento } \\
\text { comprensivo de los } \\
\text { sesgos propios. }\end{array}$ & $\begin{array}{l}\text { Aceptar las } \\
\text { explicaciones de los } \\
\text { fenómenos a su valor } \\
\text { nominal. }\end{array}$ & $\begin{array}{l}\text { Un investigador } \\
\text { considera explicaciones } \\
\text { alternativas para } \\
\text { los hallazgos que } \\
\text { las postuladas } \\
\text { anteriormente. }\end{array}$ \\
\hline 8. Interseccionalidad & $\begin{array}{l}\text { Conceptualización } \\
\text { y mediciones, } \\
\text { Acción }\end{array}$ & $\begin{array}{l}\text { La naturaleza } \\
\text { interrelacionada } \\
\text { de las categorias } \\
\text { sociales coexistentes } \\
\text { (por ejemplo, raza y } \\
\text { género) y las formas } \\
\text { de estratificación } \\
\text { social que las } \\
\text { mantienen. }\end{array}$ & $\begin{array}{l}\text { Modelo aditivo de } \\
\text { categorías sociales } \\
\text { concurrentes (por } \\
\text { ejemplo, raza y género). }\end{array}$ & $\begin{array}{l}\text { Los esfuerzos para } \\
\text { reducir las conductas } \\
\text { de riesgo de VIH entre } \\
\text { hombres diversos } \\
\text { que tienen sexo con } \\
\text { hombres abordan los } \\
\text { estereotipos raciales. }\end{array}$ \\
\hline $\begin{array}{l}\text { 9. Autocrítica } \\
\text { disciplinaria }\end{array}$ & Acción & $\begin{array}{l}\text { El examen sistemático } \\
\text { por parte de los } \\
\text { miembros de una } \\
\text { disciplina de sus } \\
\text { convenciones e } \\
\text { impactos en la } \\
\text { sociedad en general. }\end{array}$ & $\begin{array}{l}\text { Examen crítico limitado } \\
\text { de cómo las normas } \\
\text { de una disciplina } \\
\text { pueden influir en el } \\
\text { conocimiento sobre un } \\
\text { tema. }\end{array}$ & $\begin{array}{l}\text { Los investigadores } \\
\text { examinan las } \\
\text { implicaciones para la } \\
\text { investigación sobre el } \\
\text { uso de "desigualdades } \\
\text { en salud" frente a } \\
\text { "disparidades en salud" } \\
\text { frente a "inequidades } \\
\text { en salud". }\end{array}$ \\
\hline 10. Voz & $\begin{array}{l}\text { Producción de } \\
\text { conocimiento, } \\
\text { Acción }\end{array}$ & $\begin{array}{l}\text { Priorizar las } \\
\text { perspectivas de las } \\
\text { personas marginadas; } \\
\text { Privilegiar el } \\
\text { conocimiento } \\
\text { experiencial de los } \\
\text { "outsiders within". }\end{array}$ & $\begin{array}{l}\text { Privilegio rutinario } \\
\text { de perspectivas } \\
\text { mayoritarias. }\end{array}$ & $\begin{array}{l}\text { Respuestas de } \\
\text { escepticismo o enojo } \\
\text { cuando "outsiders } \\
\text { within" hablan la } \\
\text { verdad al poder. }\end{array}$ \\
\hline
\end{tabular}

Fuente: Ford y Airhihenbuwa (2010b, p. 1394, traducción nuestra)

No podemos detenernos en ellos, pero sí queremos subrayar dos aspectos de la dimensión estructural del racismo que han sido estudiados en profundidad y nos parecen importantes tanto por el valor que tienen como por la decisión con que son abordados en estos estudios.

El primero se refiere a cómo la irregularidad administrativa impacta en la salud al exponer a 
todo tipo de abuso. Al respecto, Castañeda (2017) muestra cómo el estatus migratorio afecta en múltiples dimensiones: por el miedo, el estrés, estableciendo acceso diferencial a los recursos, a las experiencias de prejuicio y violencia, a las separaciones familiares, así como al trabajo y vivienda segura. Más aun, concluye que políticas públicas como la del Affordable Care Act (ACA) incrementaron la exclusión simbólica y social de los inmigrantes al producir una nueva frontera que separó de manera más fuerte y clara a los inmigrantes no autorizados del resto del cuerpo político (Castañeda, 2017). ${ }^{6}$

Así, el efecto acumulativo de todas estas instancias lleva a una experiencia de tensión y descontento que no puede ser fácilmente superada, lo que constituye una carga extra debida exclusivamente a la vulnerabilidad propia de su posición en la sociedad (Quesada et al., 2014). A todo esto se debe añadir el riesgo de la deportación (Quesada, 2011) y el generalizado miedo que se tiene a las instituciones, que está detrás del retardo en la búsqueda de atención, aun cuando en la atención no se pregunte el estatus migratorio, aunque exista una clara y extrema necesidad de atención (Quesada, 2011, p. 391).

Los migrantes se encuentran en un estatus social racializado (Asad; Clair, 2018), es decir, en una posición social desacreditada, basada en una clasificación legal aparentemente neutral, que afecta desproporcionadamente a grupos de minorías étnico-raciales, ejerciendo una carga dispar en ellos por medio de la restricción de los derechos sociales y políticos, y de los procesos de estigmatización que afectan negativamente el estrés psicosocial y sus respuestas de afrontamiento (Asad; Clair, 2018).

El segundo aspecto se refiere a cómo las políticas dirigidas a los migrantes impactan directamente la salud produciendo un entorno sociopolítico iatrogénico (Castañeda et al., 2015; Gee; Ford, 2011; Gee et al., 2016; Morey, 2018).
En la línea de lo ya analizado por Castañeda en relación al ACA, Morey (2018) muestra que no solo ocurrió que todas las personas indocumentadas quedaron inelegibles para cualquier plan o subsidio, sino que como trabajadores rara vez calificaban para los programas de indigentes, lo que hace que el costo de la atención médica sea uno de los principales problemas que tienen que afrontar. Así, y como subrayó Castañeda (2017), la política social no solo constituye una forma de producir nuevas relaciones sociales, modos de gobierno y experiencias de cuidado, sino que en sí puede tener efectos patogénicos. Una de las formas de comprender esto es asumir que un contexto sociopolítico antinmigrante es un determinante social de la salud, que afecta principalmente a las comunidades de color, sean migrantes o nomigrantes (Morey, 2018).

Morey (2018, p. 461, traducción nuestra) señala que:

El estrés causado por la amenaza de un entorno sociopolítico que apunta específicamente a excluir y privar de derechos a grupos enteros de la población puede acumularse con el tiempo para causar un mayor "desgaste” en sus cuerpos, lo que lleva a niveles más altos de enfermedades crónicas, comportamientos de salud riesgosos y mortalidad prematura.

\section{Consideraciones finales}

Al finalizar esta revisión crítica de las tres principales aproximaciones en el abordaje del binomio migración-salud, vemos que todas las perspectivas revisadas iluminan aspectos e introducen matices en la reflexión sobre dicha relación. La revisión nos ha permitido esbozar una panorámica que, si bien no es exhaustiva, se ha mostrado reveladora de algunos de los puntos de clivaje que distinguen unas perspectivas de otras.

\footnotetext{
6 Otro tipo de frontera es la que devela Fassin (2003) al analizar las políticas humanitarias que facilitan la regularización migratoria por enfermedades graves en Francia. La crítica se sitúa en los “usos políticos del cuerpo" (Fassin, 2003, p. 49) de los inmigrantes, quienes deben exponer el sufrimiento y la enfermedad para obtener -como enfermos- la regularización previamente negada. Fassin califica de "biolegitimidad" o política de reconocimiento del ser sufriente y del cuerpo enfermo (Fassin, 2004) esta forma de gobierno de los "ilegítimos" (Fassin, 2003) que fomenta la desigualdad.
} 
La perspectiva de los DSS pone énfasis en las condiciones socioculturales desde un modelo clásico epidemiológico, que carece de un fundamento teórico que le permita reconocer un orden estructurante en la multiplicidad de causas. Esto la hace poco idónea para analizar las relaciones de poder y la relación entre las dinámicas de acumulación de capital y el proceso salud/enfermedad/atención. En efecto, hemos mostrado cómo, aun cuando son considerados ciertos aspectos materiales (las "condiciones sociales de la salud”), el análisis deshistorizado y despolitizado resulta en una objetivación que refuerza la relación que el sentido común racista establece entre los migrantes y la pobreza, la delincuencia, las drogas, la violencia, etc.

En el caso del transnacionalismo, hemos visto cómo su cuestionamiento sistemático del nacionalismo metodológico le permitió desarrollar una serie de aproximaciones teórico-metodológicas que han ampliado la comprensión de los procesos migratorios y de movilidad, en su relación con la salud. Así ha mostrado la relación de las prácticas de salud con los procesos transnacionales, la importancia de la territorialidad transnacional en la búsqueda de la atención de salud, el valor de las estructuras informales de protección social para los migrantes, así como la importancia del capital cultural trasnacional. Nuestro análisis sobre esta perspectiva se centró en mostrar cómo un uso acrítico de este último concepto puede vehiculizar ideales hegemónicos respecto de los saberes médicos de los migrantes que desconocen el valor del pluralismo médico a la vez que culturalizan el problema del acceso a la atención de salud, soslayando las causas fundamentales de las inequidades en salud (Villa-Torres et al., 2017).

En contraste con las dos perspectivas previas, la propuesta de la vulnerabilidad estructural se desarrolló al asumir decididamente la centralidad de lo estructural en la salud de los migrantes, recogiendo la tradición crítica de la medicina social. Hemos mostrado cómo, partiendo del impacto de la violencia estructural en los cuerpos, se analiza el lugar de la migración en la estructura económica capitalista; la violencia simbólica presente en la naturalización de las jerarquías sociales; y el racismo estructural, materializado en los procesos de irregularización administrativa y la producción de entornos sociopolíticos iatrogénicos.

Más allá del interés teórico que pueda tener esta panorámica, entendemos que tiene varias aristas prácticas. Primero, porque constituye el trasfondo técnico-ideológico de las políticas, programas, proyectos, diagnósticos y prácticas de atención dirigidas a la salud de los migrantes. Así, una claridad respecto de las principales coordenadas teóricas permite visualizar también los alcances que tendrán las propuestas concretas en el abordaje de las causas de la inequidad que afecta la salud de los migrantes. Segundo, porque a la luz de las críticas revisadas a la naturalización de las jerarquías sociales, así como a la reproducción del racismo estructural por medio del conocimiento científico, parece necesario problematizar en qué medida estas perspectivas teóricas contribuyen con la reproducción de la violencia simbólica ejercida contra los migrantes en el espacio de la ciencia.

Para eso es preciso, como plantea la teoría crítica de la raza, realizar un examen sistemático por parte de los miembros de las disciplinas científicas, de sus convenciones e impactos en la sociedad en general, desde la asunción de la producción ideológica del conocimiento, pues el racismo también se reproduce en la cotidianeidad de los discursos, análisis, programas y proyectos científicos. Dicha reproducción no se reduce a una cuestión de voluntades más o menos conscientes, sino que responde a los procesos de producción social de conocimiento, que siempre son procesos ideológicos, permeables a influencias, intereses, presiones económicas, en fin, a los juegos del poder.

Por supuesto la realidad no se transforma solo operando en el plano teórico, pero consideramos importante problematizar la reproducción presente en el espacio de la academia, pues es donde se sacralizan estas lecturas de la realidad, que se elevan desde el sentido común y retornan como fundamento científico de las políticas de lo cotidiano. 


\section{Referencias}

ABEL, E. K. From exclusion to expulsion: mexicans and tuberculosis in Los Angeles, 1914-1940. Bulletin of the History of Medicine, Baltimore, v. 77, n. 4, p. 823-849, 2003.

AIRHIHENBUWA, C. O.; FORD, C. L. Critical race theory: we are all others. Ethnicity \& Disease, Owings Mills, v. 28, n. 1, p. 219-222, 2018.

ASAD, A. L.; CLAIR, M. Racialized legal status as a social determinant of health. Social Science \& Medicine, Amsterdam, v. 199, p. 19-28, 2018.

BALDASSAR, L. Too sick to move: distant 'crisis' care in transnational families. International Review of Sociology, Abingdon, v. 24, n. 3, p. 391-405, 2014.

BELL, D. et al. Transnational healthcare, crossborder perspectives. Social Science \& Medicine, Amsterdam, v. 124, p. 284-289, 2015.

BONILLA-SILVA, E. Racism without racists. Oxford: Rowman \& Littlefield, 2006.

BOURGOIS, P. Conjugated oppression: class and ethnicity among Guaymi and Kuna banana workers. American Ethnologist, Hoboken, v. 15 , n. 2, p. 328-348, 1988.

BREILH, J. Las ciencias de la salud pública en la construcción de una prevención profunda: determinantes y proyecciones. In: RODRÍGUEZ, M. I. (Ed.). Lo biológico y lo social: su articulación en la formación del personal de salud.

Washington, DC: OPS: OMS, 1994. p. 63-100.

BREILH, J. La determinación social de la salud como herramienta de transformación hacia una nueva salud pública. Revista Facultad Nacional de Salud Pública, Medellín, v. 31, n. 1, p. 13-27, 2013. BURAWOY, M. The functions and reproduction of migrant labor: comparative material from Southern Africa and the United States. American Journal of Sociology, Chicago, v. 85, n. 5, p. 1050-1087, 1976.

CABIESES, B.; BERNALES, M.; MCINTYRE, A. M. La migración internacional como determinante social de la salud en Chile. Santiago: UDD, 2017.
CABIESES, B.; GALVEZ, P.; AJRAZ, N. Migración internacional y salud: el aporte de las teorías sociales migratorias a las decisiones en salud pública. Revista Peruana de Medicina Experimental y Salud Pública, Lima, v. 35, n. 2, p. 285-291, 2018.

CABIESES, B. et al. Vulnerabilidad social y su efecto en salud en Chile. Santiago: UDD, 2016.

CABIESES, B. et al. Escenario actual de la vida y salud de migrantes en la comuna de Iquique. Santiago: UDD, 2017.

CASTAÑEDA, H. Is coverage enough? Persistent health disparities in marginalised Latino border communities. Journal of Ethnic and Migration Studies, Abingdon, v. 43, n. 12, p. 2003-2019, 2017.

CASTAÑEDA, H. et al. Immigration as a social determinant of health. Annual Review of Public Health, Palo Alto, v. 36, n. 1, p. 375-392, 2015.

CASTLE, B. Public health's approach to systemic racism: a systematic literature review. Journal of Racial and Ethnic Health Disparities, New York, v. 6, n. 1, p. 27-36, 2019.

CHILE. Ministerio de Salud. Determinantes sociales en salud. Santiago, 2019. Disponível em: <https://bit.ly/39BnMvY>. Acesso em: 5 dez. 2018.

CONNELL, J. From medical tourism to transnational health care? An epilogue for the future. Social Science \& Medicine, Amsterdam, v. 124, p. 398-401, 2015.

CONTRERAS GATICA, Y.; ALA-LOUKO, V.; LABBÉ, G. Acceso exclusionario y racista a la vivienda formal e informal en las áreas centrales de Santiago e Iquique. Polis, Santiago, v. 14, n. 42, p. 53-78, 2015.

CRUSH, J.; CHIKANDA, A. South-South medical tourism and the quest for health in Southern Africa. Social Science \& Medicine, Amsterdam, v. 124, p. 313-320, 2014.

DÖRR, S.; FAIST, T. Institutional conditions for the integration of immigrants in welfare states: a comparison of the literature on Germany, France, Great Britain, and the Netherlands. European Journal of Political Research, Hoboken, v. 31, n. 4, p. 401-426, 1997. 
FAIST, T. et al. Transnational social protection: migrants' strategies and patterns of inequalities. Population, Space and Place, Hoboken, v. 21, n. 3, p. 193-202, 2015.

FASSIN, D. Santé et immigration: les vérités politiques du corps. Cahiers de l'Urmis, Nice, v. 5, p. 69-75, 1999.

FASSIN, D. Repenser les enjeux de santé autour de l'immigration. Hommes et Migrations, Paris, v. 1225, n. 1, p. 5-12, 2000.

FASSIN, D. Gobernar por los cuerpos, políticas de reconocimiento hacia los pobres y los inmigrantes en Francia. Cuadernos de Antropología Social, Buenos Aires, n. 17, p. 49-78, 2003.

FASSIN, D. Entre las políticas de lo viviente y las políticas de la vida: hacia una antropología de la salud. Revista Colombiana de Antropología, Bogotá, DC, v. 40, p. 283-318, 2004.

FEAGIN, J. Systemic racism: a theory of oppression. New York: Routledge, 2006.

FEAGIN, J.; BENNEFIELD, Z. Systemic racism and U.S. health care. Social Science \& Medicine, Amsterdam, v. 103, p. 7-14, 2014.

FORD, C. L.; AIRHIHENBUWA, C. O. Critical race theory, race equity, and public health: toward antiracism praxis. American Journal of Public Health, Washington, DC, v. 100, n. 51, p. 693-698, $2010 a$.

FORD, C. L.; AIRHIHENBUWA, C. O. The public health critical race methodology: praxis for antiracism research. Social Science \& Medicine, Amsterdam, v. 71, n. 8, p. 1390-1398, 2010b.

FRANCO, S. et al. Debates en medicina social. Quito: OPS: Alames, 1991.

GEE, G. C.; FORD, C. L. Structural racism and health inequities: old issues, new directions. $D u$ Bois Review, Cambridge, v. 8, n. 1, p. 115-132, 2011.

GEE, G. C. et al. Citizenship as privilege and social identity: implications for psychological distress. American Behavioral Scientist, Thousand Oaks, v. 6o, n. 5-6, p. 680-704, 2016.
GLICK SCHILLER, N. Theorizing about and beyond transnational processes. In: MIELANTS, E. et al (Ed.). Caribbean migration to the United States and western Europe: essays on incorporation, identity and citizenship. Philadelphia: Temple University Press, 2009. p. 18-42.

GRINESKI, S. E. Parental accounts of children's asthma care: the role of cultural and social capital in health disparities. Sociological Focus, Abingdon, v. 42, n. 2, p. 107-132, 2009.

GRINESKI, S. E. Why parents cross for children's health care: transnational cultural capital in the United States-Mexico border region. Social Theory and Health, New York, v. 9, n. 3, p. 256-274, 2011.

HICKEN, M. T. et al. Racial inequalities in health: framing future research. Social Science \& Medicine, Amsterdam, v. 199, p. 11-18, 2018.

HOLMES, S. M. "Oaxacans like to work bent over": the naturalization of social suffering among berry farm workers. International Migration, Abingdon, v. 45, n. 3, p. 39-68, 2007.

HOLMES, S. M. Structural vulnerability and hierarchies of ethnicity and citizenship on the farm. Medical Anthropology: Cross Cultural Studies in Health and Illness, Abingdon, v. 30, n. 4, p. 425-449, 2011.

HOLMES, S. M. Fresh fruit, broken bodies: migrant farmworkers in the United States. Berkeley: University of California Press, 2013a.

HOLMES, S. M. "Is it worth risking your life?": ethnography, risk and death on the U.S.-Mexico border. Social Science \& Medicine, Amsterdam, v. 99, p. 153-161, 2013 b.

HOLMES, S. M. Fruta fresca, cuerpos marchitos: trabajadores agrícolas migrantes en Estados Unidos. Quito: Abya Yala, 2016.

HORTON, S. B. Medical returns as class transformation: situating migrants' medical returns within a framework of transnationalism. Medical Anthropology: Cross Cultural Studies in Health and Illness, Abingdon, v. 32, n. 5, p. 417-432, 2013. 
IMILAN, W. Políticas y luchas por la vivienda en Chile: el camino neoliberal. Working Paper Series: Contested Cities, [S. l.], mayo 2016.

IRWIN, A.; SCALI, E. Action on the social determinants of health: a historical perspective. Global Public Health, Abingdon, v. 2, n. 3, p. 235-256, 2007.

KRIEGER, N. Epidemiology and the web of causation: has anyone seen the spider? Social Science \& Medicine, Amsterdam,

v. 39, n. 7, p. 887-903, 1994.

KRIEGER, N. Discrimination and health inequities. International Journal of Health Services, Thousand Oaks, v. 44, n. 4, p. 643-710, 2014.

LIBERONA CONCHA, N.; TAPIA LADINO, M.; CONTRERAS, Y. Movilidad por salud entre Arica y Tacna: análisis de una demanda no satisfecha y de una oferta atractiva del otro lado de la frontera. Geopolítica(s), Madrid, v. 8, n. 2, p. 253-278, 2017.

MADDEN, E. F. Cultural health capital on the margins: cultural resources for navigating healthcare in communities with limited access. Social Science \& Medicine, Amsterdam, V. 133, p. 145-152, 2015.

MÁRQUEZ COVARRUBIAS, H. Diccionario crítico de migración y desarrollo. Ciudad de México: Porrúa, 2012.

MAYRING, P. Qualitative content analysis. In: FLICK, U.; VON KARDORFF, E.; STEINKE, I. (Ed.) A companion to qualitative research. London: Sage, 2004. p. 266-269.

METZL, J. M.; ROBERTS, D. E. Structural competency meets structural racism: race, politics, and the structure of medical knowledge. AMA Journal of Ethics, Chicago, v. 16, n. 9, p. 674-69o, 2014.

MORALES-BORRERO, C. et al. ¿Determinación social o determinantes sociales? Diferencias conceptuales e implicaciones praxiológicas. Revista de Salud Pública, Bogotá, DC, v. 15, n. 6, p. 797-808, 2013.
MOREY, B. N. Mechanisms by which antiimmigrant stigma exacerbates racial/ethnic health disparities. American Journal of Public Health, New York, v. 108, n. 4, p. 46o-463, 2018.

OMS - ORGANIZACIÓN MUNDIAL DE LA SALUD. Determinantes sociales de la salud. Genebra, 2018. Disponível em: <https://bit.ly/39xogVz>. Acesso em: 5 dez. 2018.

ORGANISTA, K. C. et al. Sexual health of Latino migrant day labourers under conditions of structural vulnerability. Culture, Health \& Sexuality, London, v. 15, n. 1, p. 58-72, 2013.

ORMOND, M.; SULIANTI, D. More than medical tourism: lessons from Indonesia and Malaysia on South-South intra-regional medical travel. Current Issues in Tourism, Abingdon, v. 20, n. 1, p. 94-110, 2017.

PHELAN, J. C.; LINK, B. G. Is racism a fundamental cause of inequalities in health? Annual Review of Sociology, Palo Alto, v. 41, n. 1, p. 311-330, 2015.

PIÑONES-RIVERA, C.; MANSILLA AGÜERO, M.; ARANCIBIA CAMPOS, R. El imaginario de la horizontalidad como instrumento de subordinación: la Política de Salud de pueblos indígenas en el multiculturalismo neoliberal chileno. Saúde e Sociedade, São Paulo, v. 26, n. 3, p. 751-763, 2017.

PIÑONES-RIVERA, C.; QUESADA, J.; HOLMES, S. M. La vulnerabilidad estructural y las nuevas perspectivas en medicina social sobre la salud de los migrantes: entrevista a James Quesada y Seth M. Holmes. Salud Colectiva, Lanús, v. 15, art. e2146, 2019.

PON, G. Cultural competency as new racism: an ontology of forgetting. Journal of Progressive Human Services, Abingdon, v. 20, n. 1, p. 59-71, 2009.

QUESADA, J. No soy welferero: undocumented Latino laborers in the crosshairs of legitimation maneuvers. Medical Anthropology, Abingdon, v. 30, n. 4, p. 386-408, 2011. 
QUESADA, J.; HART, L. K.; BOURGOIS, P. Structural vulnerability and health: Latino migrant laborers in the United States. Medical Anthropology: Cross Cultural Studies in Health and Illness, Abingdon, v. 3o, n. 4, p. 339-362, 2011.

QUESADA, J. et al. "As good as it gets": undocumented latino day laborers negotiating discrimination in San Francisco and Berkeley, California, USA. City \& Society, Washington, DC, v. 26, n. 1, p. 29-50, 2014.

RYAN, W. Blaming the victim. New York: Vintage Books, 1976.

SHIM, J. K. Cultural health capital: a theoretical approach to understanding health care interactions and the dynamics of unequal treatment. Journal of Health and Social Behavior, Thousand Oaks, v. 51, n. 1, p. 1-15, 2010.

SOLAR, O.; IRWIN, A. Social determinants, political contexts and civil society action: a historical perspective on the Commission on Social Determinants of Health. Health Promotion Journal of Australia, Milton, v. 17, n. 3, p. 180-185, 2006.

SONTAG, S. La enfermedad y sus metáforas. Madrid: Taurus, 2003.

STONINGTON, S. D. et al. Case studies in social medicine: attending to structural forces in clinical practice. The New England Journal of Medicine, Boston, v. 379, p. 1958-1961, 2018.

TIJOUX, M. E.; CÓRDOVA, M. Racismo en Chile: colonialismo, nacionalismo, capitalismo. Polis, Santiago, v. 14, n. 42, p. 7-13, 2015.
TIJOUX, M. E.; PALOMINOS, S. Aproximaciones teóricas para el estudio de procesos de racialización y sexualización en los fenómenos migratorios de Chile. Polis, Santiago, v. 14, n. 42, p. 247-275, 2015.

VILLA-TORRES, L. et al. Transnationalism and health: a systematic literature review on the use of transnationalism in the study of the health practices and behaviors of migrants.

Social Science \& Medicine, Amsterdam, v. 183, p. 70-79, 2017.

VIRUELL-FUENTES, E. A.; MIRANDA, P. Y.; ABDULRAHIM, S. More than culture: structural racism, intersectionality theory, and immigrant health. Social Science \& Medicine, Amsterdam, v. 75, n. 12, p. 2099-2106, 2012.

WILLIAMS, D. R.; COLLINS, C. Racial residential segregation: a fundamental cause of racial disparities in health. Public Health Reports, Thousand Oaks, v. 116, n. 5, p. 404-416, 2001.

WIMMER, A.; GLICK SCHILLER, N. Methodological nationalism and beyond: nation-state building, migration and the social sciences. Global Networks, Hoboken, v. 2, n. 4, p. 301-334, 2002.

WIMMER, A.; GLICK SCHILLER, N. Methodological nationalism, the social sciences, and the study of migration: an essay in historical epistemology. The International Migration Review, Thousand Oaks, v. 37, n. 3, p. 576-610, 2003. WHO - WORLD HEALTH ORGANIZATION. Health of migrants resetting the agenda: report of the and global consultation. Genebra, 2017.

\section{Contribución de los autores}

Piñones-Rivera trabajó en la investigación, metodología, análisis y redacción del texto. Liberona contribuyó con la investigación y la redacción de la versión final. Leiva trabajó en la redacción de la versión final del artículo.

Recibido: 10/08/2020

Aprobado: 09/09/2020 